\title{
Natural resource modelling: Allowing for inconstant probability of detection and frequency measures of violation within dynamic deterrence fishery models
}

\author{
Sana Abusin; Rashid Hassan; Greg Hertzler
}

\begin{abstract}
Sana Abusin is a $\mathrm{PhD}$ candidate, at the Centre for Environmental Economics and Policy in Africa (CEEPA), Department of Agricultural Economics, Extension and Rural Development, University of Pretoria, 0002. Pretoria, South Africa. Corresponding author

Email: sn_abusin@hotmail.com
\end{abstract}

Sanaa.abusin@gmail.com

Tel: +27762262012

Rashid Hassan is director of the Centre for Environmental Economics and Policy in Africa (CEEPA), Department of Agricultural Economics, Extension and Rural Development, University of Pretoria, 0002. Pretoria, South Africa.

G. Hertzler is an Extraordinary Professor at the Centre for Environmental Economics and Policy in Africa (CEEPA), University of Pretoria and Associate Professor at the University of Sidney, Australia.

\begin{abstract}
:
The dynamic deterrence model (DDM) represents an analytical framework widely used for analyzing compliance with fishery regulations aiming for sustainable fishery management. However, applications of the DDM have so far been limited to the case of constant probability of detection that assumes independence of the length of time to detection. This paper modifies the DDM to allow for more flexible and broader specification by introducing, for the first time, two important variables to the supply of offences function, namely evasion activity and enforcement efforts and accommodating inconstant probability of detection specifications. Determinants of probability of detection are specified and important potential extensions of the modified DDM are
\end{abstract}


discussed in this paper. The relative magnitude of the effects of each of these determinants on probability of detection, however, remains an important empirical question that requires further investigation for prioritization of policy actions.

Keywords: Dynamics; Illegal fishing; Profit functions; proportional hazard rate

\section{Introduction}

In natural resource modelling, dynamic models have been developed to analyze allocation of resources over time (inter-temporal allocation decisions). The dynamic deterrence model (DDM) is one example of an analytical framework widely used for analyzing compliance with fishery regulations aiming for sustainable fishery management. The two periods DDM, first developed by Davis (1988) postulates that violators seek to maximize their expected discounted profit over two periods, where in the first period, offenders gain from illegal activities until they get caught and pay a fine, after which they will engage only in legal activities in the second period.

The applications of DDM have so far been limited to the case of constant probability of detection that assumes independence of the length of time to detection (Akpalu, 2008/2009; Davis, 1988; Leung, 1991). The factors that determine the probability of detection function also lack clarity in explanation in this model. As probability of detection is considered to be a salient issue of compliance, better understanding of this function is very important. There are good reasons to believe that probability of detection could be time-dependent. The Cox's proportional hazard function allows this hypothesis 
to be accommodated and provides a better explanation of factors influencing probability of detection.

Inconstancy of probability of detection could be influenced by factors beyond the control of violators. For instance, the probability of the violator being caught is small in an artisanal setting but higher when the fishing industry is highly commercialised. Other sources of inconstancy of probability of detection include violator's aversion to the risk of being arrested, which varies over time because of age or simply luck or any factor that is assumed to make the hazard rate change over time. Three important modifications to the standard DDM are suggested in this paper to allow for a more flexible specification and better explanation of the factors determining compliance with regulations.

The paper proceeds by defining the extensions to the existing analytical framework of compliance models in next section. Section three formally develops the adapted model and its extensions. Section four concludes the paper with suggestions for potential further extensions of the DDM.

\section{Extending the current analytical framework}

This study adapts the standard DDM introducing three important modifications: allowing for random detection time, relaxing the assumption of constant probability of detection and employing frequency rather than intensity of violation as a measure of violation rate. 


\subsection{Allowing for random time of detection}

Although the DDM segregates violation rate into two periods, namely, before and after getting caught, all previous literature using this model formulates the choice problem to be optimised over an infinite time horizon. The transition between the two periods is therefore not clear. This study assumes that time of detection is a random variable that defines the end of the first period and the start of the second period, which then extends to infinity in period two. Splitting the two periods would then result in an easier distinction between the violation and compliance periods within the decision time horizon.

\subsection{Inconstant probability of detection}

A key contribution of this study is that it adapts the DDM to allow for inconstancy of probability of detection by employing the Cox proportional hazard function to represent it. The probability of detection is thus defined as a function of the multiple of two terms, namely, a constant individual characteristics function and a time-variant hazard function.

\subsection{Measuring violation rate by frequency instead of intensity of violation}

The rate of violation in the DDM is measured either by frequency of violation (number of times a fisher violates regulations) in static models (Eggert and Lokina 2010; Hatcher and Gordon 2005, Sutinen and Kuperan 1999; King and Sutinen 2010 and Furlong 1991) or intensity of violation, which is measured by the proportion of illegal catch compared to 
the total catch in dynamic models (Akpalu 2008). Intensity of violation may be applicable in the context of developed countries but it is highly unlikely to work well in developing countries where fishers can easily escape detection and where monitoring and enforcement of regulation is weak.

\section{The DDM for compliance}

The DDM adapted for this study assumes that violators enjoy incremental profits from violation in the first period until detected at time $\tau$, which marks the end of the first period. The offender is then punished at the end of the first period and begins to behave legally thereafter (to compare between the standard and modified DDMs see annex A).

Suppose that the goal of an individual fisher who violates mesh size regulation is to maximize his/her profit from the two periods before and after being caught. The profit from fishing illegally in the first period is defined as $\pi(m)$. The violation rate $m$ should accordingly be positive $(m>0)$ during the first period and zero through period two. We assume that the gain from violation increases with $m$ at a decreasing rate, i.e. $\frac{\partial \pi(m)}{\partial m}>$ $0 \& \frac{\partial^{2} \pi(m)}{\partial m^{2}}<0$. Assuming that after being caught, the fisher will only fish legally (i.e. $m=0$ ) and gets a constant profit net of the fine $F$, we define profit in the second period as $\pi(n)$. A perfect selectivity assumption is hold in this case, i.e. nets with the legal mesh size can harvest only mature stock, and those with the illegal mesh size can harvest both mature and immature stock.

Moreover, we assume that in absolute terms, illegal fishing is more profitable than legal fishing. 


$$
\pi(m)>\pi(n)
$$

As stated earlier, we assume that in the first period, the violator will fish until he/she is caught at a random time $\tau$ in the future, given that she has never been caught before. The second period starts from the random time $\tau$ when the fisher is caught and required to pay a fine $F$, which incorporates a fixed amount of money and the cost of the illegal net, which will be seized immediately. The violator's inter-temporal expected profit is accordingly given by:

$J(m, n)=\max _{m} E\left\{\int_{0}^{\tau} e^{-\delta \tau} \pi(m) d t+\int_{\tau}^{\infty} e^{-\delta \tau} \pi(n) d t-e^{-\delta \tau} F\right\}$

Where $J($.$) is the value function, \mathrm{E}$ is the mathematical expectation , $\delta$ is the discount rate, $\pi(m)$ and $\pi(n)$ are, respectively, the profit from illegal and legal fishing, $\tau$ is the random time when the second period starts (the time the fisher is caught and required to pay the fine $F$ ).

Two factors motivate the formulation of this model. First, in most of the developing countries, the management regime is known as "regulated open access" where fishers can catch as much as they can as long as obeying some regulations such as mesh size regulations as considered in this paper.

Secondly, although violators do generally recidivate, because of poverty and difficulty in buying an illegal net (expensive), we assume that the fisher is highly unlikely to 
recidivate, following literature (Akpalu 2008). This implies that this model is closer to developing countries situations.

Like previous models (Davis 1988; Leung 1991; Akpalu 2008/2009) we assume that the violator does not know the exact time of detection, but has information about the probability distribution of time of detection. Accordingly, we specify a continuous distribution of time of detection $\tau$ with a probability density function - $\operatorname{pdf} \mathrm{g}(\tau)$ and cumulative density function - cdf $\mathrm{G}(\tau)$ such that $\mathrm{g}(\tau)=\mathrm{dG}(\tau) / \mathrm{d} \tau$, where $0 \leq \tau \prec \infty$. Then, the violator's objective function is to maximize the expected discounted profits from fishing illegally in the first period and legally after getting caught, subject to the survival time (the details of this calculation are presented in Annex A).

$J()=.\max _{m}\left\{\frac{\pi(m)}{\delta}-\left[\frac{\pi(m)-\pi(n)+F}{\delta}\right] E e^{-\delta \tau}\right\}$

The interpretation of this equation is important. The first term is the discounted expected benefit from illegal fishing for an infinite time horizon, and the second and third parts (between brackets) illustrate the penalty that the violator should pay when getting caught, which includes lost net gains from illegal fishing (difference between legal \& illegal fishing profits) plus the fine imposed $F$.

The use of expected utility or profit is motivated by the fact that the gain from violation is not guaranteed, since violators might get caught at any time. All previous specifications used both density function and cumulative functions to represent probability distribution 
of detection time. Here we use the mathematical expectation with respect to the density function.

The goal of the violator is then to maximize equation 3, subject to probability of detection. We explain in the following section the new specification of inconstant probability of detection introduced to the DDM for the first time.

So far in the literature, the probability of detection function has been modelled as constant over time, while some attempts have considered influences of probability of detection to vary only with violation rate $m$ but still independent of time (Leung 1991). Accordingly influences of important factors were not explicitly entered in the supply of offence function of the deterrence model. This study modifies previous specifications of the hazard function to allow for better understanding of influences of factors determining noncompliance by relaxing key assumptions.

To relax the assumption of constancy of probability of detection; we adopt Cox's proportional hazard model (CXPHM). This model is mostly used in survival analysis (Cox 1972) commonly applied to analysis of data in different fields of sciences such as medicine, environmental health, criminology, and marketing (Jenkins 2005; Lee and Go 1997). The said model is particularly popular in medical sciences when measuring the survival of patients with serious diseases. This study used the CXPHM to define survival time, which is linked to the hazard rate (probability of detection) in order to define the density function with the following general expression: 
$\mathrm{H}(\mathcal{T}, m, v, n)=\mathcal{B}(m, v, N) h(\mathcal{T})$, or

$\operatorname{Pr}(\mathcal{T}, m, v, n)=\mathcal{B}(m, v, N) h(\mathcal{T}) \quad 0 \leq \mathcal{B} \leq 1$ and $\frac{d \beta}{d m}>0$

Previous DDM specifications assumed probability of detection to be a function of violation rate $m$ (i.e. $\operatorname{Pr}(m)$ ). Equation 4 above introduces key modifications in the standard DDM previous formulations. The right-hand side of equation 4 includes two hazard functions: the first $\beta($.$) is the individual-specific hazard function, which does not$ depend on $\tau$, whereas the second (the baseline hazard function) $h(\tau)$ depends on time but not on $\beta$ factors. The latter function is the one that determines whether the hazard rate is constant, decreasing or increasing, explained further below. Equation 4 implies that probability of detection is linearly related to individual hazard function $\beta($.$) , which is$ expected to increase with the crime rate $(m)$ and decrease with enforcement $(N)$ and evasion activities ( $v$ ) as cited in the literature (Charles et al. 1999). The important implication of the above CXPHM specification is that the baseline hazard function can accommodate constant and inconstant time dependence.

Most of the literature on survival analysis using the Cox proportional hazard rate assumes an exponential distribution because of ease and convenience in computation. However, this assumption does not have a realistic survival time in real life (Bender et al. 2003). The most frequently used distribution for survival time is the Weibull distribution (Lee and Go, 1997). Using this type of distribution allows for the three situations of constant, decreasing or increasing probability of detection. 
We accordingly specify the following proportional hazard rate function:

$$
h(\tau)=\frac{g(m, v, N, \mathcal{T})}{1-G(m, v, N, \mathcal{T})}
$$

Applying some calculations and rearrangements of terms to link the proportional hazard rate and the pdf, both formulas are equated. Then the density function as a function of the proportional hazard rate is given as follows (see Annex B for details):

$g(\tau, m, N, v)=\mathcal{B}(m, v, N) h(\mathcal{T}) \mathrm{e}^{-\mathcal{B}(m, v, N) \int_{0}^{t} h(\mathcal{T}) d \tau}$

Incorporating this into the value function specified in equation 3 the violator's maximization problem can then be written as:

$J()=.\max _{m}\left[\frac{\pi(m)}{\delta}\right]-\left[\frac{\pi(m)-\pi(n)+F}{\delta}\right] \int_{0}^{\infty} g(\mathcal{T}, m, \mathcal{v}, N) e^{-\delta \mathcal{T}} d t$

Therefore, the net gain from violation is the expected discounted value of illegal fishing minus the discounted expected penalty. The last term represents the discounted density of time of detection, which is a function of explanatory variables (m, $v, \mathrm{~N}$ ), determining the hazard rate and rate of violation. For simplicity, the last term of equation (7) will be replaced by the following formula throughout the text:

$D(\tau, m, N, v)=\int_{0}^{\infty} g(\mathcal{T}, m, v, N) e^{-\delta \mathcal{T}} d t$ 
According to the standard DDM, Probability of detection has a concave relation with $m$ (Davis, 1988) which implies that

$\frac{\partial D}{\partial m}>0$

Substituting equation (8) in the value function, gives the following form:

$J=\max _{m}\left[\frac{\pi(m)}{\delta}\right]-\left[\frac{\pi(m)-\pi(n)+F}{\delta}\right] D(\tau, m, N, v)$

The optimal level of violation is obtained from the first order conditions by differentiating the objective function with respect to $m$ to decide on the optimal amount of $m$ that maximizes the profit through the optimal path. For the violator to maximise her/his profit, equation (9) is assumed to satisfy concavity conditions (annex C):

$\frac{\partial J}{\partial m}=0=\left[\frac{\partial \pi(m) / \partial m}{\delta}\right][1-D]-\left[\frac{\pi(m)-\pi(n)+F}{\delta}\right] \frac{\partial D}{\partial m}$

$\frac{\partial^{2} J}{\partial m^{2}}>0$

Denoting the first order condition in equation 10 by K, we have:

$K=\left[\frac{\partial \pi(m)}{\partial m}\right][1-D]-[\pi(m)-\pi(n)+F] \frac{\partial D}{\partial m}$ 
Solving 12 for optimal frequency of violation level $\left(m^{*}\right)$ and differentiating $m^{*}$ with respect to its arguments, we can perform comparative static analysis of the influences of introduced factors on the frequency of violation, particularly punishment severity $F$, evasion efforts $v$, and enforcement measures $N$.

\section{Calculation of the effect of each factor on the rate of violation}

Invoking the implicit function theorem for function $\mathrm{K}\left(\mathrm{m}^{*}(\alpha), \alpha\right)$, where $\alpha$ is a vector of the set of arguments in the model and $m$ is at its optimal level $m^{*}$ (to simplify presentations we hereafter omit the $*$ ), the following hold for each argument $\alpha_{j}$ at the optimum level $m *$ :

$\frac{d K}{d \alpha_{j}}=\frac{\partial K}{\partial m} * \frac{\partial m}{\partial \alpha_{j}}+\frac{\partial K}{\partial \alpha_{j}}=0 \quad$ Or in other words: $\quad \frac{\partial m}{\partial \alpha_{j}}=-\frac{\partial K}{\partial \alpha_{j}} / \frac{\partial K}{\partial m}$

Using the above, we can derive the following comparative static' results

\subsection{Influence of fine severity $(F)$ on frequency of violation}

$\frac{\partial K}{\partial F}=-\frac{\partial D}{\partial m}<0$

For result 14 to have the expected negative sign supporting the theoretical expectation that higher deterrence will reduce violation, $\frac{\partial D}{\partial m}$ has to be positive, implying that probability of detection (density function of detection time $D$ ) must be higher with more frequent violations (higher $m$, or $\frac{\partial D}{\partial m}>0$ ). This seems to support plausible hypotheses about compliance with regulation. 


\subsection{Influence of evasion efforts ( $v$ ) on frequency of violation}

$$
\frac{\partial K}{\partial V}=-\frac{\partial}{\partial V}\left[\frac{\partial \pi(m)}{\partial m} D+[\pi(m)-\pi(n)+F] \frac{\partial D}{\partial m}\right]<0
$$

It is implicit that gains from illegal fishing $\pi(\mathrm{m})$ increase with more violation (i.e. $\partial \pi / \partial \mathrm{m}$ $\geq 0)$ and that violation brings higher gains $(\pi(m)-\pi(n) \geq 0)$. Since $F$ and $D$ are both positive, and as implied by result 15 above $\frac{\partial D}{\partial m}>0$ (higher violation rates increase probability of detection), then result 16 suggests that investment in evasion efforts is inversely related to violation rate (i.e. a negative sign on 15). This result is consistent with theoretical hypotheses (Charles et al, 1999) which suggests that evasion activity decreases gains from violation since it increases the cost of attempting safer violation and hence lowers the need for more frequent violations (reducing the need for more frequent attempts to violate). This is the first time that such hypothesis is tested as allowed by the current specification of the DDM and also invokes an important implication in terms of the trade-off between higher evasion efforts (v) and higher rates of violation (frequency $m$ ) in fishers’ decision making, to which we will return later.

\subsection{Influence of enforcement measures $(\mathrm{N})$ on frequency of violation}

$$
\frac{\partial K}{\partial N}=-\frac{\partial}{\partial N}\left[\frac{\partial \pi(m)}{\partial m} D+[\pi(m)-\pi(n)+F] \frac{\partial D}{\partial m}\right]<0
$$

The same arguments made under result 15 hold and hence the negative sign of result 16, implying that an increase in enforcement discourages violation. 


\subsection{Influence of discount rate measures on frequency of violation}

$$
\frac{\partial K}{\partial \delta}=-\frac{\partial \pi(m)}{\partial m} \frac{\partial D}{\partial \delta}-[\pi(m)-\pi(n)+F] \frac{\partial}{\partial m}\left[\frac{\partial D}{\partial \delta}\right]>0
$$

Since the discount rate and the function $\mathrm{D}$ are negatively related (see annex $\mathrm{D}$ ), the overall sign is positive, implying that increase in the discount rate increases frequency of violation. This result has been previously reported in the literature (Akpalu, 2008).

\section{Concluding remarks}

In this paper, we modify the DDM in two specific ways to allow for a more flexible and broader specification. First, we introduce time as a random variable in the distribution of the density function to accommodate inconstant probability of detection by using a proportional hazard rather than the survival hazard used in existing literature. Second, we employ frequency rather than intensity of violation as a measure of violation rate. These extensions introduce two variables into the supply of offences function, which are evasion activity $v$ and enforcement $N$, a major feature of this paper and a contribution to existing literature on compliance.

The method of comparative static was used to derive important analytical results that are in line with theory and prior results reported in the literature. It shows that violation decreases with higher fines, and greater evasion and enforcement levels. Derived comparative static' results suggest important potential modifications to the DDM. For 
instance, it is clear that the profit from illegal activity depends on both evasion efforts (v) and violation rate (frequency $m$ ). This implies that the model can be reformulated to have two control variables, $m$ and $v$. In such a case, the violator will seek to choose the optimal combination of these two variables. Thus, a useful extension of the DDM is to consider the trade-off between these two choices and possibilities of substitution.

The modified DDM can be used to empirically simulate influences of key determinants of compliance under alternative formulations. For example, the discounted density function that is used to model probability of detection in this paper can assume different distribution functions, which allows for the three possibilities of constant, increasing and decreasing probability of detection employing the Weibull distribution. Therefore, simulation and sensitivity analyses can be performed and outcomes compared under the three situations. This may be implemented through maximising the deterrence model in equation 9 subject to the proportional hazard equation 4 on any optimization algorithm.

The relative magnitude of the effects of each of the determinants on probability of detection, however, remains an important empirical question that requires further investigation for prioritization of policy actions. For example, a regression analysis of the determinants of probability of detection can be used by applying the Weibull proportional hazard regression model, using the programme SAS as explained by the literature in the medical and criminology fields. This is done by choosing the appropriate independent variables assumed to affect this probability (in the case of this paper, these are the variable that measured enforcement, avoidance activities and violation rate) and another 
variable that is dependent on time, such as age, as the baseline function (Bender et al., 2003; Lee and Go, 1997; Bodenhorn and Price, 2009; Maddan et al., 2008). This kind of data is rarely found especially in developing countries.

Applications of the model developed in this study are not limited to the fishery case but can be generalised to management and regulation of other natural resources such as exploitation of common property forest, water and grazing lands and hunting of wildlife.

\section{References}

W. Akpalu [2008], Individual Discount Rate and Regulatory Compliance in a Developing Country Fishery, Environ. Devel. Econ. 13, 591-606.

W. Akpalu [2009], A dynamic model of mesh size regulatory compliance, workings paper no 123, Farmingdale state college.

A. T. C. Charles, R. L. Mazany and M. L. Cross [1999], the Economics of Illegal

Fishing: A Behavioral Model. Marine Resource Econ. 14, 95-110. 
M. Davis ICHAEL [1988], Time and punishment: an inter-temporal model of crime, J. Polit. Economy 96, 383-90.

H. Eggert and Lokina [2010], Regulatory compliance in Lake Victoria fisheries, Environ. Devel. Econ. 1-21 Cambridge University Press.

W. J. Furlong [1991], The Deterrent Effect of regulatory Enforcement in the fishery, Land Econ. 67(1), 116-129.

A. Hatcher and D. Gordon [2005], further investigation into the factors affecting compliance with UK fishing quotas, Land econ. 81, 71-86.

S. P. Jenkins [2005], Survival Analysis, Unpublished manuscript, Institute for Social and Economic Research, University of Essex, Colchester, UK, [Online], Available : ॥ http://www.iser.essex.ac.uk/teaching/degree/stephenj/ec968/pdfs/ec968lnotesv6.pdf.

D. M. King and J.G. Sutinen [2010], Rational noncompliance and the liquidation of Northeast ground fish resources, Marine Pol. 34(1), 7-21.

J. Sutinen and K. Kuperan and [1999], Blue Water Crime: Deterrence, Legitimacy, and Compliance, In Fisheries, Law and soci. revi. 32(2), 309-338.

F. S. Lueng [1991b], How to make the fine fit the corporate crime? An analysis of static and dynamic Optimal punishment theories, J. Public Econ. 45, 24356.

E. Lee, O. Go [1997], Survival analysis in public health research. Annu Rev of Public Health, 18: 105-134. 
D. Cox [1972], Regression Models and Life Tables (with Discussion). J. Roy. Statistical Society, Series B 34:187—220.

R. Bender, T. Augustin, \& M. Blettner [2003], Generating Survival Times to Simulate Cox Proportional Hazards Models. online available at http://epub.ub.uni-muenchen.de. Paper 338. Corresponding author: Ralf Bender, PhD, statician. University of Bielefeld.

S. Maddan, J. T. Walker and J. M.Miller [2008], “Does Size Really Matter?: A Reexamination of Sheldon's Somatotypes and Criminal Behavior.” Soc. Sci. J.45, $330-344$.

H. Bodenhorn; G. Price [2009], Crime and body weight in the nineteenth century: was there a relation between brawn, employment opportunities and crime? Workings paper 15099. http://www.nber.org/papers/w15099. Cambridge. 


\section{Annex A. Modifying the standard DDM}

(Comparing the standard and modified model)

The standard DDM postulates maximizing the following value function:

$$
v(.)=\int_{0}^{\infty} e^{-\delta t} \pi(m)(1-G(t))+\pi(n) \quad G(t)-R F g(t) d t
$$

Where $v($.$) is the violation rate, \pi(m)$ and $\pi(n)$ are, respectively, profit from illegal and legal fishing; $G(t)$ and [1-G(t)] are, respectively, probability of being caught and not caught; $\mathrm{F}$ is the fine imposed on illegal fishing; $\mathrm{R}$ is the probability that a fisher is fined given that s/he is detected; and $\delta$ is the discount rate. Defining probability of being detected (hazard rate) $\operatorname{Pr}(\mathrm{m})$ ) as:

$$
\begin{aligned}
& \operatorname{Pr}(m)=\frac{g(t)}{1-G(t)} \\
& \quad=\frac{-d(1-G(t) / d t}{1-G(t)} \\
& \operatorname{Pr}(m)=\frac{-d \ln (1-G(t) / d t}{d(t)}
\end{aligned}
$$

Integrating both sides, we reach:

$$
\begin{aligned}
& \int_{0}^{\mathrm{t}} \operatorname{Pr}(m) d t-\ln (1-G(t)) \\
& \ln \{1-\mathrm{G}(\mathrm{t})\}=-\int_{0}^{\mathrm{t}} \operatorname{Pr}(m) d t \text { hence }\{1-\mathrm{G}(\mathrm{t})\}=\exp -\int_{0}^{\mathrm{t}} \operatorname{Pr}(m) d t
\end{aligned}
$$


Then the values of the density and cumulative functions are:

$$
\begin{gathered}
\{1-G(t)\}=e^{-\int_{0}^{\infty} \operatorname{Pr}(m) d t} ;\{G(t)\}=1-e^{-\operatorname{Pr}(m) t} \operatorname{and}\{g(t)\} \\
=\operatorname{Pr}(m) e^{-\operatorname{Pr}(m) t}
\end{gathered}
$$

Substituting the values of $g(t)$ and $G(t)$ in equation and assuming that all other variables are constant over time, we get the value function of each violator

$v()=.\frac{\pi(m)-R F \operatorname{Pr}(m)}{\delta+\operatorname{Pr}(m)}+\frac{\pi(n)}{\delta}$

This is the same value function alternatively expressed in this paper as:

$J()=.\max _{m}\left\lfloor\left[\frac{\pi(m)}{\delta}\right]-\left[\frac{\pi(m)-\pi(n)+F}{\delta}\right] E e^{-\delta \mathcal{T}}\right\rfloor$

Where, $\boldsymbol{E}\left\{\boldsymbol{e}^{-\boldsymbol{\delta} \mathcal{T}}\right\}=\int_{0}^{\infty} g(.) \boldsymbol{e}^{-\boldsymbol{\delta} \mathcal{T}} d \boldsymbol{t}$

The main modification we introduce is in the specification of $g($.$) as we make g vary over$ time and influenced by individual hazard function factors such as evasion and enforcement activities. This defines a new value function:

$J()=.\max _{m}\left[\frac{\pi(m)}{\delta}\right]-\left[\frac{\pi(m)-\pi(n)+F}{\delta}\right] \int_{0}^{\infty} g(\mathcal{T}, m, v, n) e^{-\delta \mathcal{T}} d t$ 
This section shows all the steps for the integration to calculate the expected net present value of illegal gain using the modified two times dynamic deterrence model.

Where $\pi(m)=\pi_{m \text { and }} \pi(n)=\pi_{n}$,

$$
\begin{aligned}
& J(.) \quad=\max _{m} E \int_{0}^{\mathcal{T}} e^{-\delta} \pi_{m} d t+E \int_{\mathcal{T}}^{\infty} e^{-\mathcal{S}} \pi_{n} d t-e^{-\delta \mathcal{T}} F \frac{1}{\delta} \\
& =\max _{m} E \int_{0}^{\mathcal{T}} e^{-\delta} \pi_{m} d t+E \int_{\mathcal{T}}^{\infty} e^{-\mathcal{S}}\left[\pi_{m}-\pi_{n}\right] d t-e^{-\delta \mathcal{T}} F \frac{1}{\delta} \\
& =\max _{m} E \int_{0}^{\mathcal{T}} e^{-\delta} d t+E\left[\pi_{m}-\pi_{n}\right] \int_{\mathcal{T}}^{\infty} e^{-\delta} d t-e^{-\delta \mathcal{T}} F \frac{1}{\delta} \\
& =\max _{m} E\left\{\left.\pi_{m} \frac{-e^{-\delta \delta}}{\delta}\right|_{t=0} ^{t \rightarrow \infty}-\left.\left[\pi_{m}-\pi_{n}\right] \frac{-e^{-\delta t}}{\delta}\right|_{t=\tau} ^{t \rightarrow \infty}-e^{-\delta \mathcal{T}} F \frac{1}{\delta}\right\} \\
& \text { 0. } \max _{m} E\left\{\frac{\pi_{m}}{\delta}-\frac{\left[\pi_{m}-\pi_{n}\right]}{\delta} e^{-\delta \mathcal{T}}-e^{-\delta \mathcal{T}} F \frac{1}{\delta}\right\} \\
& \text { [ }=\max _{m} E\left\{\frac{\pi_{m}}{\delta}-\frac{\left[\pi_{m}-\pi_{n}+F\right]}{\delta} e^{-\delta \mathcal{T}}\right\} \\
& \text { [] }=\max _{m}\left\{\frac{\pi_{m}}{\delta}-\frac{\left[\pi_{m}-\pi_{n}+F\right]}{\delta}\right\} E\left\{e^{-\delta \mathcal{T}}\right\} \\
& \text { 0 }=\max _{m}\left\{\frac{\pi_{m}}{\delta}-\frac{\left[\pi_{m}-\pi_{n}+F\right]}{\delta}\right\} \int_{0}^{\infty} g(\tau, m, N, v) e^{-\delta \mathcal{T}} d \tau
\end{aligned}
$$

Equation (A-1) is the discounted net present value of a fisher who violates the first period (first term) plus the gain from the second period (second term). After in between calculation and integration, we reached equation A-2, which give us the exact expected discount profit from violation, the first term is the gain from violation and the second term is the amount of penalty that the fisher gets after being caught (the difference between legal and illegal plus fine) the outcome will be the pure gain from violation. 
In equations A-3, we insert the value of the expectation parameter, which is the net present value of the time of detection.

\section{Annex B. Calculating the Probability density (the relations between the density function and proportional hazard rate)}

This is straightforward calculation to get the proportional density function $g($.$) from the$ hazard formula and inserts the final results in the maximisation equation.

$$
\operatorname{Pr}(\mathcal{T}, m, v, n)=\mathcal{B}(m, v, n) h(\mathcal{T})
$$

With the survival function given by:

$$
\begin{aligned}
& h(\tau)=\frac{g(\tau, m, n, v)}{1-G(\tau, m, n, v)} \\
& =\frac{\frac{d G(\tau, m, n, v)}{d \tau}}{1-G(\tau, m, n, v)} \\
& =\frac{-d(1-G(\tau, m, n, v)) / d \tau}{1-G(\tau, m, n, v)}
\end{aligned}
$$




$$
=\frac{-d \ln (1-G(\tau, m, n, v)) / d \tau}{d \tau}
$$

Integrating both sides we get

$$
\begin{gathered}
\int_{0}^{\mathcal{T}} \mathrm{h}(\tau, m, n, v) d \tau=-\ln \{1-G(\tau, m, n, v)\} \\
-\int_{0}^{\mathcal{T}} \mathrm{h}(\tau, m, n, v) d \tau=\ln \{1-G(\tau, m, n, v)\}
\end{gathered}
$$

Hence

$$
1-G(\tau, m, n, v)=\exp \left(-\int_{0}^{t} h(\tau, m, n, v) d \tau\right)
$$

Which can written as

$$
1-G(\tau, m, n, v)=\mathrm{e}^{\left(-\int_{0}^{t} \mathrm{~h}(\tau, m, n, v) d \tau\right)}
$$

If the periodic harvest in this model is assumed to be constant overtime then

$$
\begin{aligned}
& 1-G(\tau, m, n, v)=\mathrm{e}^{\left(-\int_{0}^{t} \mathrm{~h}(\tau, m, n, v) d \tau\right)} \\
& -G(\tau, m, n, v)=\mathrm{e}^{(-\mathcal{B}(m, v, n) h(\mathcal{T})} \\
& G(\tau, m, n, v)=1-\mathrm{e}^{-\mathcal{B}(m, v, n) \int_{0}^{t} h(\mathcal{T}) d \tau}
\end{aligned}
$$


And,

$g(\tau, m, n, v)=\mathcal{B}(m, v, n) h(\mathcal{T}) \mathrm{e}^{-\mathcal{B}(m, v, n) \int_{0}^{t} h(\mathcal{T}) d \tau}$

Substituting fro $g(\tau, m, n, v)$ in the value function we obtain:

$J=\max _{m}\left[\frac{\pi(m)}{\delta}\right]-\left[\frac{\pi(m)-\pi(n)+F}{\delta}\right] \int_{0}^{\infty} g(\mathcal{T}, m . v, n) e^{-\delta \mathcal{T}} d t$.

\section{Annex C}

\section{Calculation of the first and second order condition}

For the violator to maximise his profit the value function should be concave.

$$
\frac{\partial J}{\partial m}=0=\left[\frac{\partial \pi(m) / \partial m}{\delta}\right][1-D]-\left[\frac{\pi(m)-\pi(n)+F}{\delta}\right] \frac{\partial D}{\partial m}
$$

$$
\begin{aligned}
\frac{\partial^{2} J}{\partial m^{2}} & =\left(\frac{1-D}{\delta} \frac{\partial^{2} \pi}{\partial m^{2}}\right)-\left(\frac{\partial^{2} D}{\partial m^{2}} \frac{\pi(m)-\pi(n)+F}{\delta}+\frac{\partial D}{\partial m}\left(\frac{\partial \pi / \partial m}{\delta}\right)\right) \\
& =\frac{1}{\delta}\left((1-D) \frac{\partial^{2} \pi(m)}{\partial m^{2}}-\frac{\partial \pi(m)}{\partial m} \frac{\partial D}{\partial m}\right)-\left(\frac{\partial^{2} D}{\partial m^{2}}\left(\frac{\pi(m)-\pi(n)+F}{\delta}\right)\right)<0
\end{aligned}
$$




\section{Annex (D)}

Relation between probability of detection and the discount rate

The relation between probability of detection and the discount rate is calculated as follows:

$D(\tau, m, N, v)=\int_{0}^{\infty} g(\mathcal{T}, m, v, n) e^{-\delta \mathcal{T}} d t$

Then

$\frac{\partial D}{\partial \delta}=-\int_{0}^{\infty} \tau g(.) e^{-\delta \mathcal{T}} d t$ 